\title{
La renovación urbana en la ciudad ideal renacentista de Leonardo da Vinci
}

\section{Urban renewal in the city ideal renaissance of Leonardo da Vinci}

\author{
DAVID HidAlgo GARcía \\ Profesor Colaborador Doctor \\ Departamento de Expresión Gráfica Arquitectónica y en la Ingeniería. Universidad de Granada \\ dhidalgo@ugr.es
}

Recibido: 20/06/2015

Aceptado: 10/09/2015

\begin{abstract}
Resumen
Durante las primeras décadas del siglo XV surgió en Italia un grupo de arquitectoshumanistas que aspiraron a desarrollar una nueva concepción de urbe que rompiera con la tradición llevada a cabo durante la Edad Media. Estas nuevas ideas pretendían una renovación urbana sin precedentes, dirigidas fundamentalmente a la regulación de los espacios públicos. Entre las propuestas realizadas destaca la de Leonardo da Vinci (1452-1519), quien elaboró estudios arquitectónicos y urbanísticos de un excelente nivel que han sido olvidados por la sociedad. De todos estos estudios, destaca su propuesta de città idéale que por su innovador planteamiento de renovación urbana debería haber supuesto un punto de inflexión en la morfología urbana de las ciudades. Una revolución sin precedentes que modificó la forma de entender el concepto de ciudad en el Renacimiento y en los siglos posteriores. Todo ello en una disciplina en la que nunca ha sido reconocido como tal.
\end{abstract}

Palabras clave

Renacimiento, urbanismo, morfología urbana, renovación urbana. 


\begin{abstract}
During the first decades of the fifteenth century arose in Italy a group of architectshumanists who aspired to develop a new concept of city that break with tradition held during the Middle Age. These new ideas intended unprecedented urban renewal mainly aimed at regulating public spaces. Among the proposals highlights the Leonardo da Vinci (1452-1519), who produced architectural and urban renewal of an excellent standard that have been forgotten by society studies. In all these studies, emphasizes its proposal città ideale for its innovative approach to urban renewal should have been a turning point in the urban morphology of cities. An unprecedented revolution that changed the way we understand the concept of city in the Renaissance and in later centuries. All this in a discipline that has never been recognized as such.
\end{abstract}

\title{
Keywords
}

Renaissance, urbanism, urban morphology, urban renewal.

Referencia normalizada: HIDALGO GARCÍA, DAVID (2015): “La renovación urbana en la ciudad ideal renacentista de Leonardo da Vinci". Arte y Ciudad. Revista de Investigación, no 8 (octubre), págs. 59-82. Madrid. Grupo de Investigación Arte, Arquitectura y Comunicación en la Ciudad Contemporánea, Universidad Complutense de Madrid.

Sumario: 1.- Introducción. 2.- Formación de los arquitectos del Renacimiento. 3.- La renovación urbana en la ciudad ideal de Leonardo. 4.- Conclusiones. 5.- Bibliografía.

\section{Introducción}

En la cultura renacentista, la proyección de la ciudad constituyó un elemento primordial de gran importancia. El desarrollo y estudio de esta adquirió una singular relevancia dentro de la sociedad ya que desde mediados del siglo XIII, se inició en las ciudades un proceso de decoro urbano (Fuente, 1991) y de regulación de los espacios, tanto públicos como privados. Las intervenciones se desarrollaron sobre elementos individuales, aunque en algunas casos, estas afectaban a la globalidad de la urbe (Franchetti, 1985). El proceso continuó mediante la elaboración de una serie de normas y reglamentaciones ${ }^{1}$ que controla-

${ }^{1}$ El objetivo fundamental de la normativa y reglamentación aprobada era el decoro de las ciudades. El decoro se entendía no solo como la estética de las edificaciones sino también en relación a la moralidad y las costumbres, tales como: higiene en la edifica- 
ban las actividades y edificaciones ${ }^{2}$ de la ciudad. Finalizó en los primeros decenios del siglo XV con la aspiración de llegar a una nueva concepción de urbe, conocida con el nombre de città idéale 3 .

Para Vittorio Franchetti, las líneas según las cuales se desarrolló el proceso de renovación de las ciudades del Renacimiento fueron cuatro. La primera línea consistió en la mejora del decoro urbano de las urbes. La segunda línea estaba determinada por la búsqueda de nuevos tipos de construcción, los cuales exigían, en algunos casos, cambios en el trazado de calles, plazas o espacios abiertos. La tercera línea fue la relacionada con el problema de las ampliaciones de las ciudades. Estas generalmente se produjeron fuera de los sistemas de fortificación y supusieron auténticos barrios o guetos de clases sociales bajas. La última línea, fue la de la búsqueda teórica de la completa renovación urbana, es decir, la creación de la ciudad ideal (Franchetti, 1985).

A partir del siglo XV y sobre todo en el ámbito italiano, puede advertirse que esas exigencias fueron dirigidas a la regulación de los espacios. Esta circunstancia geográfica ha despertado una especial atención dentro de los estudiosos de la historia del urbanismo. Durante el inicio del periodo renacentista se modificaron los modos, estilos de vida, las costumbres sociales y el pensamiento de la sociedad. Esto supuso simultáneamente una modificación en las actitudes y comportamientos respecto a los usos del territorio y a la organización de la ciudad (Franchetti, 1985).

Según los datos históricos de los que se tiene constancia, en el siglo XV se desarrolló en Italia una cultura urbanística de larga y antigua tradición, de la que se han podido obtener algunos tratados y teorías importantes. La forma de pensar y estudiar las artes (la pintura, la escultura y la arquitectura) del Renacimiento, se trasladó a la forma de proyección y generación de las nuevas ciudades. Estas constituyeron un elemento primordial, sobre todo a mediados del

ción, desplazamiento de las actividades ruidosas y malolientes a las zonas exteriores, equipamientos urbanos para favorecer el almacenamiento y seguridad frente a incendios (Arévalo, 2000).

2 En la Storia di Milano de Bernardino Arluno, publicada en 1530, se hace referencia al modo en el que los edificios de la ciudad de Milán eran embellecidos con decoraciones monocromáticas en sus fachadas (Pedretti, 1988: 74).

${ }^{3}$ Sobre el concepto de utopía e ideal ver (Calatrava, 2010). 
siglo $\mathrm{XV}$, cuando apareció un grupo de arquitectos humanistas italianos que defendieron la teoría de que la ciudad era un organismo urbano y por lo tanto, planificable en todas sus partes (Cantone, 1978). Todas las zonas o espacios de la urbe debían tener un carácter unitario y constituir un todo de la urbe. De esta forma, cada elemento de la ciudad (plaza, calle, edificio, etc), dejó de ser un elemento independiente para pasar a una parte de ese organismo unitario que se pretendió fuera parte de la ciudad. Además, y una vez desarrollada la ciudad como conjunto unitario, su objetivo fue el de perdurar a lo largo del tiempo (Franchetti, 1985). Evelio Moreno va mucho más allá y afirma: “La città idéale de los renacentistas italianos es la alternativa al paraíso. [. . . Este es sustituido por una città como comunidad de vida y pensamiento" (Moreno, 1991: 9-13). En los inicios, la città idéale fue pensada como un elemento global de bienestar para los ciudadanos y no como un conjunto de calles y viviendas dotadas de unas infraestructuras desconocidas hasta ese momento (Puppi, 1973).

Por tanto, se desarrolló una línea en la que la ciudad se podía asimilar a un organismo antropomórfico cuyos máximos exponentes fueron los arquitectosFrancesco di Giorgio Martini, Baldassarre Peruzzi y Filarete ${ }^{4}$. A propósito de las ciudades, la introducción del Tratati di Architettura Ingegneria e Arte Militare de Francesco di Giorgio indica:

Teniendo la ciudad juicio, medida y forma del cuerpo humano, circunferencias y divisiones las describiré con precisión. En primer lugar ha de saberse que, extendiendo en el suelo el cuerpo humano, puesto un hilo del ombligo a la extremidad y girándolo se obtendrá la circunferencia. Similarmente se pueden hacer diseños cuadrados y angulares. Consecuentemente puede observarse cómo el cuerpo tiene todas las divisiones y miembros con perfecta medida y circunferencias, lo mismo en la ciudad y otros edificios (Muratore, 1980: 124).

En la línea opuesta, se encuentran arquitectos como León Battista Alberti, Leonardo da Vinci, Filarete o Vincenzo Scamozzi para los que la ciudad no debía tener relación con el cuerpo humano y su función no dejó de ser otra que la de albergar y satisfacer las necesidades de las personas. En algunos casos no solo necesidades físicas sino también psíquicas o de bienestar.

${ }^{4}$ La primera idea en relación a la proporción de las ciudades a partir del cuerpo humano fue tenida en cuenta por Luca Pacioli en su tratado Divina proportione. 
A modo de resumen, se puede indicar que el nacimiento de estas propuestas teóricas de renovación urbana mediante la creación de ciudades ideales, surgen como consecuencia de:

1. Defensa Militar: Durante la Edad Media los precarios sistemas defensivos de las ciudades eran suficientes para resistir el asalto producido mediante las armas de fuego. Tras la invención del cañón se exigió que los sistemas defensivos sufrieran un gran cambio para poder resistir los asaltos (Croix, 1960). Por este motivo, se diseñaron ciudades resistentes al asedio externo ${ }^{5}$. Tanto a nivel de sistemas defensivos y de distribución de la morfología urbana, como a nivel de capacidad de autosuficiencia durante un largo periodo en caso de asedio. Hasta la mitad del siglo XVI, las fortalezas, los sistemas defensivos y las ciudades se ubicaban dentro del campo de la arquitectura, por lo que eran tratados por arquitectos, artistas o ingenieros militares. A partir de ese momento se produjo una división en dos ramas dentro de la arquitectura: civil y militar (Vera, 2010).

2. Corrección de deficiencias urbanas: Las nuevas propuestas tienden a contar con espacios libres, acondicionamiento de calles e instalaciones de salubridad. Dichos planes llegaron a unos niveles excepcionales con ciertos arquitectos. Por ejemplo, los estudios de las condiciones de soleamiento de las calles y viviendas realizados por Pietro Cataneo y Leonardo da Vinci, o en los estudios de ventilación de viviendas realizados por León Battista.

\section{Formación de los arquitectos del Renacimiento.}

El Renacimiento trajo consigo un cambio en la arquitectura y en los arquitectos, que pasaron del anonimato a marcar en cada trabajo su estilo personal. Este desconocimiento en las autorías arquitectónicas, por ejemplo, en las catedrales, es denominado por Martínez de Aranda "el secreto de las catedrales" (recogido por Arévalo, 2000: 64), en alusión al silencio llevado a cabo sobre la construcción de este tipo de edificaciones. Circunstancia apoyada también por Lotz: "Nunca hemos encontrado ningún dibujo hecho por un arquitecto italiano que

\footnotetext{
${ }^{5}$ Sobre la influencia de las armas de fuego en el diseño de las ciudades, consultar (Vagnetti, 1974: 267).
} 
se pudiera fechar con certeza antes del año 1460 aproximadamente. Los primeros dibujos datan del último cuarto del Siglo XV" (Lotz, 1985: 1-3). Federico Arévalo defiende que el no encontrar ningún dibujo no significa que no existieran. Antes del Renacimiento, las representaciones gráficas eran realizadas en soportes tales como yeso, tablillas de madera, o el propio suelo, elementos más económicos en comparación con las hojas de papiro (Arévalo, 2000).

Estos secretos estaban motivados por el método de trabajo gremial implantado en la Edad Media y vigente en el Renacimiento. Consistían en un modelo de asociación económica que englobaba a personas que ejercían una misma profesión u oficio y que se regían por unas ordenanzas o estatutos. Se iniciaron en la Edad Media y funcionaron hasta la aparición de las fábricas en el inicio de la Revolución Industrial. Sus objetivos eran los de garantizar el trabajo y las condiciones económicas de los trabajos realizados y la regulación de los sistemas de aprendizaje y formación de los futuros profesionales (Moreno, 2001). El pintor Cennino Cennini nacido en 1370 cuenta como era el proceso de formación de un artesano-artista:

En primer lugar habrás de pasar como mínimo un año practicando el dibujo en la pizarrilla; luego estarás con el maestro en el taller hasta que hayas aprendido todas las ramas que componen nuestro arte. Luego comenzarás a preparar las pinturas, aprenderás a cocer la cola, a moler el yeso y el método de imprimar con él; aprenderás a repujar, a dorar, a granear bien; durante seis largos años. Y luego pasarás a las pruebas prácticas en pintura, a trazar decorados, a hacer telones, a ejercitarse en la pintura mural por seis años más (citado en AA.VV., 2013: 9).

Los gremios se estructuraban en tres niveles: maestro-oficial-aprendiz. Estos gremios pudieron adoptar distintas denominaciones según el lugar donde se encontraran, así en Venecia se conocían como matricole, en Padua fraglie, en Lombardía consolati, en Roma università. En el resto de países europeos también se utilizó un término distinto, así en Francia se conocían como maitrises, o en España gremios (Vagnetti, 1974).

La arquitectura no estaba considerada como un oficio independiente y por tanto no contaba con un sistema de aprendizaje específico. Por lo general, el arquitecto del Renacimiento había adquirido la formación a través de otra disciplina, sea por ejemplo, la escultura, la pintura o la orfebrería. De esta forma, si tras demostrar su valía en el arte estudiado se adquirían conocimientos en 
aritmética, geometría y teoría de las proporciones el artista podía convertirse en arquitecto. Según la clasificación de las artes de la Edad Media, estas podían ser artes liberales o artes mecánicas. Las primeras se enseñaban en las Universidades y las segundas a través de los gremios. La pintura y la escultura estaban consideradas artes mecánicas y por tanto solo se podía aprender a través de los maestros (AA.VV., 2013).

La primera referencia histórica de la que se tiene constancia en relación a la formación o cualificación de un arquitecto fue realizada por Vitruvio en su obra De Architectura, recopilatorio de temas relacionados con la arquitectura, distribuido en diez libros y dedicado al emperador Augusto. Para Vitruvio:

La arquitectura es una ciencia que surge de muchas otras ciencias, y adornada con muy variado aprendizaje; por la ayuda de que un juicio se forma de esos trabajos que son el resultado de otras artes. La práctica y la teoría son sus padres. La práctica es la contemplación frecuente y continuada del modo de ejecutar algún trabajo dado, o de la operación mera de las manos, para la conversión de la materia de la mejor forma y de la manera más acabada. La teoría es el resultado de ese razonamiento que demuestra y explica que el material forjado ha sido convertido para resultar como el fin propuesto. Porque el arquitecto meramente práctico no es capaz de asignar las razones suficientes para las formas que él adopta; y el arquitecto de teoría falla también, agarrando la sombra en vez de la substancia. El que es teórico así como también práctico, por lo tanto construyó doblemente; capaz no solo de probar la conveniencia de su diseño, sino igualmente de llevarlo en ejecución [...] Y así comunicaré que sea un hombre ingenioso y de buen entendimiento aparejado para aprender. Pues la habilidad sin estudiar; o el aprender sin tener natural para ello nunca lo sacará un buen maestro. Y que sea listo y leído, liberal, gran tracista, entienda prospectiva, sepa contar. Tenga noticias de historias y antigüedades, tenga algunos principios de filosofía natural y astrología y no ignorante de medicina. Perciba música y haya pagado las ordenanzas de las leyes del edificar que se suelen guardad en los pueblos [....] Primeramente ha de ser estudioso y tenga noticia de lo que ha pasado y lo tenga asentado y notado [....] Ayuda en gran manera la geometría a este arte del edificar. Porque de tener hecha la mano al muncho del compás se viene con facilidad a saber trazar y sacar moldes y planta forma (Vitruvio, 1999: 10).

En el Renacimiento, el arquitecto León Battista Alberti establece en el proemio del libro I, capítulo I de su tratado De re aedificatoria: 
El arquitecto será aquel que con un método y un procedimiento determinados y dignos de admiración haya estudiado el modo de proyectar en teoría y también de llevar a cabo en la práctica cualquier obra que, a partir del desplazamiento de los pesos y la unión y el ensamble de los cuerpos, se adecue, de una forma hermosísima, a las necesidades más propias de los seres humanos (Alberti, 1991: 29).

En esta definición no se hace referencia a una formación universitaria, que el propio Battista recibió, sino a las cualidades y conocimientos que debía tener la persona que profesara la arquitectura, no importando si fueron adquiridos a través de maestros o en la Universidad.

Francesco di Giorgio indicó en su Trattato di archittectura civile e militare, Quarto Trattato denominado il Templi:

Ma se l'architettore non ha prespicase e sigulare ingegno e invenzione, none aspetti mai perfettamente tale arte esercitar potere, imperò che l'architettura è solo una sittile immaginzazione concetta in nella mente la quale in nell'opra si manifesta. Anco è da notare che d'ogni e ciascuna cosa non si può la ragione assegnare, perchè lo ingegno consiste più un nella mente e in nello intelletto dell 'architettore che in iscrittura o disegno, e molte cose accade in fatto le quali l'architetto overo opratore mai pensò. E imperò bisogna che esso architettore sia pratico e sciente, memorioso e che abbi letto e veduto molte cose e alla cosa che segue essare preparato. E non siccome molti arroganti e presunuosi i quali nelli errori fondati sono e per forza della lingnia loro el falso dimostrando el mondo hanno corrotto. E per volere in parte le ragioni d'essa architettura siccome seguirà discriverò. In prima è da sapere due cose essare grandemente necessarie: frabica e eciocinazio. La frabica è circa all'uso e pensiero dell'opare. Raciocinazio è demostrare le cose, innanzi che fabbricate sieno, con proporzionata ragione. E siccome dice Vetrivio al architetto ingegno e dottrina a lui bisogna, perchè lo ingegno senza dottrina o la dottrina senza igegno l'artefice perfetto far non può (Giorgio, 1967: 36-37)'.

${ }^{6}$ El arquitecto que no es perspicaz y de ingenio e invención singular no podrá ejecutar perfectamente tal arte, porque la arquitectura es solo un pensamiento sutil concebido en la mente que se manifiesta en la obra. Por esto es de notar que de toda y cada cosa no se pueda enseñar la razón, porque el ingenio consiste más en la mente y en el intelecto del arquitecto que en la escritura o en el diseño, y muchas cosas suceden de hecho las cuales ni el arquitecto ni el operario jamás pensaron. $Y$, por tanto, es necesario que el arquitecto sea práctico y sabio, que tenga memoria y que haya leído y visto muchas cosas para estar preparado ante las eventualidades. En primer lugar ha de saber dos cosas que son muy necesarias: obra y raciocinio. La obra es referente al uso y pensamiento de la acción. Raciocinio es mostrar las cosas antes que sean 
Para Vincenzo Scamozzi en su obra L'idea dell architettura universale (1615):

El arquitecto contempla en su mente los aspectos matemáticos y los hechos de la naturaleza en su magnificencia, de modo que se necesita para preparar materiales como para construir edificios ornamentales de 5 formas. Esto, sin embargo, no es elaborado como el autor, sino como un filósofo (Scamozzi, 1615, libro I, cap. VIII: 24. Trad. David Hidalgo)

Girolamo Maggi también estableció en su tratado Della fortificatione delle citta, las características que debe tener un arquitecto:

Se requiere que el entendido en Arquitectura sepa de dibujo, tenga experiencia en el arte de la medición, entienda la perspectiva, sepa aritmética, conozca mucha historia, posea la filosofía, entienda la música, la medicina, las leyes (citado en Mrakic, 2013: 103. Trad. David Hidalgo).

Resulta interesante como ningún autor de los indicados hace referencia a la necesidad de haber cursado estudios reglados para ser arquitecto. Casi todos reseñan las cualidades y conocimientos que debe tener un arquitecto en relación con la materia. En el Renacimiento no se exigían tales requisitos y por este motivo los arquitectos generalmente eran artesanos y pertenecían a gremios específicos. Por lo tanto, habían adquirido los conocimientos de la arquitectura a través de sus maestros o mediante la consulta de tratados y códices.

Leonardo da Vinci está incluido dentro de ese grupo de autodidactas del Renacimiento que adquirieron sus conocimientos a través de maestros y de la consulta de los tratados de otros autores. Así, su estancia en el taller de Andrea de Verrochio en la ciudad de Florencia, o sus trabajos conjuntos con Francesco di Giorgio ${ }^{7}$, Donato Bramante ${ }^{8}$ o Luca Pacioli ${ }^{9}$ pueden ser considerados relacio-

hechas con proporcionado juicio. Y como dice Vitruvio el arquitecto precisa ingenio y doctrina porque el ingenio sin doctrina o la doctrina sin ingenio no pueden hacer al artífice perfecto. (Trad. Vera, 2010: 30-31).

${ }^{7}$ Francesco di Giorgio Martini se había trasladado a Milán en el año 1490 para participar en el concurso convocado por la Venerable Fabrica del Duomo de Milán para la realización de su cimborrio. Allí conoce a Leonardo y se inicia un periodo de trabajo conjunto. ${ }^{8}$ Donato Bramante nació en 1443 en Monte Asdrualdo, cerca de Urbino. Se trasladó a Milán en torno al año 1480, donde erigió varias iglesias según el canon de forma y significado de la antigüedad, coincidiendo en la urbe con Leonardo en los trabajos de restauración de Santa María delle Grazie. 
nes entre maestro-aprendiz. "Leonardo adquiere gran parte de su conocimiento arquitectónico tras un minucioso estudio del tratado de arquitectura militar y civil de Francesco di Giorgio" (Taglialagamba, 2010: 7. Trad. David Hidalgo).

El interés de Leonardo por los problemas y soluciones arquitectónicos y urbanísticos se manifiesta claramente en sus Códices (Pedretti, 1988), especialmente en el Manuscrito $B^{10}$, correspondiente al periodo de 1483-1489 cuando se encontraba en Milán a las ordenes de Ludovico Sforza. En su interior se puede apreciar una especie de manual para el arquitecto donde se describe la organización de una ciudad completa denominada città idéale.

\section{La renovación urbana en la ciudad ideal de Leonardo.}

En el año 1483, Leonardo se trasladó a la ciudad de Milán con el objetivo de ponerse a las órdenes de Ludovico Sforza. Por entonces, la urbe contaba con aproximadamente 120.000 habitantes, muralla perimetral de protección, calles estrechas y tortuosas que seguían un plan radial y condiciones de insalubridad elevadas. Leonardo vivió allí el desorden, el ruido, las calles estrechas, tortuosas, la inmundicia y el hedor de una ciudad típicamente medieval. El propio Leonardo dejó constancia de estas condiciones mediante una alegoría en forma de fábula que dejó reflejada en el Códice Atlántico, folio 175v-a:

Una pietra, novamente per l'acque scoperta, di bella grandeza, si stava sopra un certo loco rilevata, dove terminava un dilettevole boschetto, sopra una sassosa strada, in compagnia d'erbette, di vari fiori di diversi colori ornata, e vedea la

${ }_{9}^{9}$ Luca Pacioli, fraile franciscano que con tan solo 19 años enseñaba aritmética, geometría y contabilidad. Leonardo lo conoció en 1498 en la ciudad de Milán e intentó aprender de él todo lo relacionado con la geometría. Se estima que tuvieron una estrecha amistad y prueba de ello es que Pacioli publicó en 1498 el tratado De Divina proportione que incluye 60 ilustraciones de poliedros realizados con acuarelas por Leonardo.

${ }^{10}$ El manuscrito B se encuentra incluido dentro de la colección denominada Manuscritos de Francia. Su nombre se debe a que fueron robados en el año 1795 por Napoleón Bonaparte y trasladados al Institut de France en Paris. Estos se encuentran compuestos por 12 códices identificados con las letras de la A a la M. El Manuscrito B fue escrito por Leonardo cuando tenía 30 años de edad, y fue presentado ante Ludovico Sforza como ingeniero militar. Está compuesto por 100 páginas con unas dimensiones de $23 \mathrm{~cm} \times 16 \mathrm{~cm}$. Fue iniciado por la página 100 y escrito como el resto de códices de derecha a izquierda. 
gran somma delle pietre che nella a sé sottoposta strada collocate erano. Le venne desiderio di là giù lasciarsi cadere, dicendo con seco: Che fo io qui con queste erve? Io voglio con queste mie sorelle in compagnia abitare. E giù lassatasi cadere, infra le desiderate compagne finì suo volubile corso. E stata alquanto, cominciò a essere da le rote de'carri, dai piè de'ferrati cavalli d de'viandanti in continu travaglio; chi la volta, quale la pestava, alcuna volta si levava alcuno pezzo, quando stava coperta del fango o sterco di qualche animale, e invano riguardava il loco donde partira d era, inel loco della soletaria e tranquilla pace. Cosi accade a quelli, che della vita soletaria e contemplativa vogliano venir a abitare nelle città, infra i pooli pieni d'infiniti mali (citado en Firpo, 1963: 64-65) ${ }^{11}$.

Entre los años 1484 y 1485, la ciudad sufrió una de las peores epidemias de peste de todo el Renacimiento, que la convirtió rápidamente en un problema político y social del Ducado. Fue tal la envergadura que la población disminuyo en $2 / 3$ partes, resultando un total de 40.000 habitantes.

Ludovico encargó a Leonardo el estudio de los motivos que habían originado aquella epidemia tan dramática y lo animó a que presentara un proyecto de ciudad alternativo para evitar que volviera a ocurrir. Un programa de renovación urbanística que mejoraría las condiciones higiénicas, de habitabilidad y que corregiría los males de la ciudad. Así, Leonardo y tras asegurarse que la consecuencia de aquella epidemia eran las condiciones de insalubridad de la ciudad, presentó su propuesta de renovación urbana, incluida dentro de la línea de ciudades ideales del Renacimiento. La mayoría de los autores establecen que Leonardo realizó una sola propuesta de ciudad ideal, sean los casos de Pedretti o Taglialagamba. Por el contrario, Luigi Firpo establece que fueron dos

${ }_{11}$ Hubo una vez una hermosa piedra, de gran tamaño, que la acción del agua había dejado al descubierto, y que vivía en compañía de hierbecitas y de flores de diversos colores. Y viendo la gran cantidad de piedras colocadas en aquella calle, le vino el deseo de dejarse caer hacia abajo, diciendo para sí: ¿Qué hago yo aquí con estas hierbas?, yo quiero vivir en compañía de mis hermanas. $Y$ dicho esto se dejó caer calle abajo, acabando su voluble carrera entre sus deseadas compañeras. Pero muy pronto comenzarían sus penas al sufrir las ruedas de los carros y las pisadas de los herrados caballos y de los viandantes; unos le hacían dar la vuelta, otros la hollaban, $y$, de vez en cuando, saltaba un guijarro; otras veces quedaba cubierta por el fango o el estiércol de algún animal. Y en vano miraba una vez y otra al lugar de donde había partido, a aquel lugar de su solitaria y tranquila paz. Así les ocurre a quienes, desde la vida solitaria y contemplativa, quieren ir a vivir a las ciudades, entre gentes llenas de infinitos males (citada en Franchetti, 1985: 567). 
las propuestas realizadas. Firpo las nombra como: città fluviale y pianni selezionati dall altro (Firpo, 1963: 68). Se procede a realizar un análisis por separado de las propuestas que establece Firpo para obtener una comparativa con las posibles similitudes y diferencias que pudieran existir.

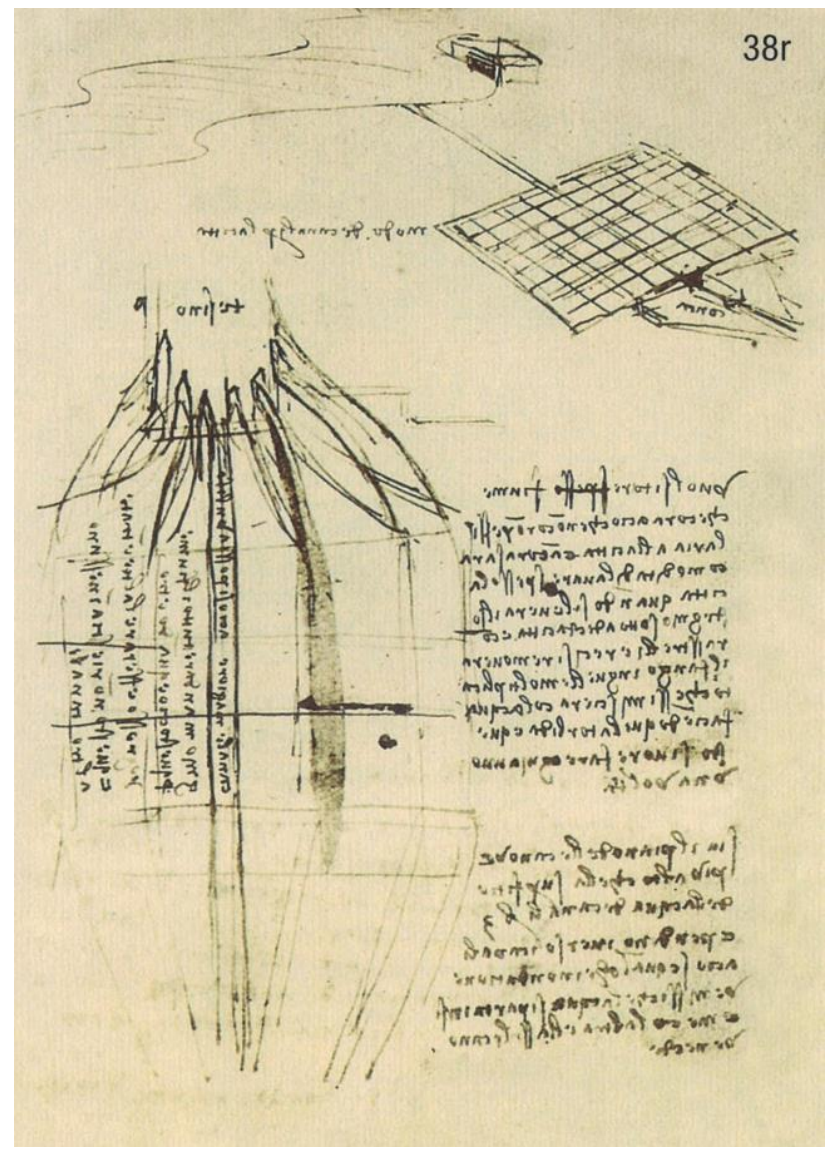

Fig. 1. Leonardo da Vinci. Ciudad ideal. Manuscrito B, folio 38r.

La città fluviale se encuentra reflejada en el Manuscrito $B$, folio 38r (véase fig. 1), escrito entre los años 1487 y 1489 en la ciudad de Milán, y ofrece una densa concentración de maravillas que van desde la historia, la arquitectura militar y civil, máquinas, catedrales, fortalezas, y finalmente artefactos misteriosos. Se trata de un libro de secretos, (militares y civiles), que revela las verdaderas aptitudes de Leonardo en la arquitectura y el urbanismo. En la parte superior derecha del folio, aparece un trazado de canales en forma de retícula. El río principal llega a la segregación en retícula, distribuye a todos los canales 
y vuelve a salir a su cauce, que sigue la dirección de entrada alejándose de la ciudad. Aparece la anotación de Leonardo Modo décanali per la città (Firpo, 1963: 68). En la parte de la izquierda, aparece un haz de canales paralelos cruzados por otros alineados perpendicularmente al eje del río. Este garantizaría la circulación del agua por la red de canales.

Canale magiore a ciò si possi a un bisogno mandare tutto il fiume per questo, cioè quando è troppo grosso, e serare l'altre entrate; e questo non riescia in nessuno altro canale [.....] Invece l’ampia dicitura sulla destra chiarisce el concetto ispiatore: Vuolse torre fiume che corra, a ciò che non corompessi l'aria della città; e ancora sarà comodità di lavare spesso la città (Firpo, 1963: 68).

En la descripción de Leonardo solo hace referencia a la utilidad limpiadora que desarrolla el río dentro de la urbe. No indica ninguna otra cuestión con respecto a esta ciudad, ni en relación a sus calles, viviendas, palacios, espacios públicos, etc. Esta ciudad no aparece reflejada en ninguna otra lámina de sus Manuscritos. Por tanto, puede interpretarse como una distribución subterránea y no como una urbe en sí, coincidiendo de esta manera con la idea del resto de autores en relación a que se trataba de una sola ciudad. Además, Leonardo escribía sus códices de derecha a izquierda y desde el final del libro hacia el principio. La ciudad fluvial viene representada en el folio 38r, mientras que el resto de documentación de esta, aparece en los siguientes folios, es decir, con numeración más baja. Por tanto, puede interpretarse como una distribución subterránea y no como una urbe en sí, coincidiendo de esta manera con la idea del resto de autores en relación a que se trataba de una sola ciudad completa y distribuida en dos niveles.

La otra propuesta de ciudad ideal, la que el autor Firpo establece como pianni selezionati dall altro (Firpo, 1963: 68), también se encuentra desarrollada en el Manuscrito B. El número de folios donde aparece es elevado en comparación con los estudios urbanísticos de la ciudad fluvial. La propuesta completa quedó reflejada en los siguientes folios: 37r, 37v, 36r, 16r y 15v, y consistía en un conjunto de diez ciudades iguales con capacidad para 30.000 personas ${ }^{12}$ cuya mor-

\footnotetext{
${ }^{12}$ E trarra'di dieci città cinquemila case con trentamila abitazioni, e disgregherai tanta congregazione di popolo (Firpo, 1966: 66) (Trazar diez ciudades de cinco mil casas con treinta mil habitantes y descomponerla en congregaciones). El autor Simoncini es el único que introduce una variable al final del texto en relación al destino de esas nuevas vivien-
} 
fología urbana sigue la forma de una planta cuadrada o rectangular, atravesada por un sistema de canales, (similar a un campamento militar romano). Estos canales están alimentados por el río Ticino, por lo que el agua fluiría eficientemente y permitiría no solo el suministro y el traslado de los habitantes y mercancías de una parte de la ciudad a otra, sino también la evacuación de aguas residuales hacia los ríos y mares (Pedretti, 1988).

El propio planteamiento de Leonardo supuso una importante revolución dentro del campo de la renovación urbana y el higienismo ${ }^{13}$, ya que desarrolló un esquema desconocido hasta ese momento ${ }^{14}$. Esta propuesta le otorgaba a Leonardo un excelente conocimiento de la planificación del territorio que le permitió anticiparse a su época. El único autor que propuso algo similar fue Baldassarre Peruzzi en su Trattato de Architettura Militare escrito entre los años 1527 y 1530. Leonardo destacó e influyó en los sistemas de representación cartográfica que se venían desarrollando hasta ese momento. Algunos autores consideran sus obras como el origen de la cartografía moderna debido a que terminó con el sistema de vista oblicua que venía desarrollándose y a su técnica de resaltar las altitudes mediante distintas tonalidades (Zollner, 2007). Una de las representaciones más destacables es el mapa de la ciudad de Imola, realiza-

das: "prevalentemente agrícola" (Simoncini, 1974: 76). Esta circunstancia podría entenderse como que las nuevas ciudades propuestas por Leonardo tendrían un uso agrícola. Posiblemente, y en consonancia con el resto de autores, se refiere a que como el desmesurado aumento de población de la ciudad de Milán se debía a la inmigración desde las zonas rurales, es evidente que las nuevas viviendas construidas tendrían un uso de albergar a esta nueva población. Véase (Simoncini, 1974: 70-80).

${ }^{13}$ Gaetana Cantone (1978: 78) indica en relación a las ciudades satélite: Pretenden dar cabida a la ciudadanía inmigrante del país, sirven para asegurar la lealtad de los campesinos que "conectan y obligan" con el príncipe, pero esta circunstancia no debe alterar el equilibrio de la ciudad central existente; para esto hay que organizar adecuadamente temprano el contexto regional. La inclusión de la expansión del núcleo en la región es uno de los puntos más importantes de los proyectados (Trad. D. Hidalgo). Por tanto, Cantone indica que las ciudades satélite de Leonardo servirían para alojar a la población inmigrante que provenía del campo. Para realizar correctamente esta distribución es necesario organizar el desarrollo territorial mediante una previa planificación urbana.

${ }^{14} \mathrm{El}$ autor Sisi lo confirma comentando: En la planificación urbana profunda, en el aspecto moderno de la técnica y el arte tiende a la organización de una región geográfica territorial y humana (Sisi, 1953: 25. Trad. D. Hidalgo). 
do mediante una técnica desarrollada por el arquitecto León Battista Alberti en el año 1450 y titulada Ludi Matematici. Mediante esta técnica se puede obtener la representación gráfica de la ciudad partiendo de un punto central y midiendo las distancias a los edificios y calles.

Por tanto, Leonardo pensó en una ciudad abierta, sin murallas de protección. La muralla impide el crecimiento horizontal y una vez agotadas las zonas de crecimiento se inicia un proceso de crecimiento vertical que genera edificaciones de mayor altura. Leonardo obvia este elemento constructivo de gran coste para el gobierno y para los ciudadanos ${ }^{15}$ proponiendo la primera y única ciudad del Renacimiento sin muralla de protección, adelantándose 400 años a sus predecesores.

Se estaría proponiendo un volumen de viviendas y población importante que garantizarían grandes ciudades en comparación con las ciudades del Renacimiento. Estas cifras fueron siglos atrás empleadas por Ebenezer Howard para las ciudades satélite en su propuesta de ciudad jardín. Leonardo propuso diez ciudades, todas ellas iguales tanto en forma física como en forma política y dependientes de la ciudad de Milán. Howard propuso en el siglo XIX ciudades satélite ubicadas anexas a la ciudad principal y conectada con la principal mediante vías de comunicación.

Las vías de comunicación y los canales, (véase fig. 2), se encuentran distribuidos en dos niveles, el superior con vías abiertas y soleadas para el paso de personas. El inferior, oscuro y cerrado, con uso para el tránsito de mercancías y animales, ubicado a nivel de los patios inferiores de las viviendas, establos y de la zona ruidosa y comercial de la ciudad (Pedretti, 1985).

Los canales circulan bajo las viviendas lo que permite la descarga directa de los desperdicios al mismo y la posibilidad de llevar mediante barcas las mercancías hasta el nivel inferior de las viviendas. Cuenta con túneles transversales que permiten la conexión con los almacenes de los edificios. Los viales del nivel superior se encuentran a una altura de 3,60 metros respecto al nivel del canal,

\footnotetext{
${ }^{15}$ Es la primera propuesta de ciudad a dos niveles de la que se tiene constancia. Posteriormente se han producido otras propuestas pero todas ellas en los siglos XIX y XX, sean las propuestas de Eugene Henard para la ciudad de París o las teorías de Le Corbusier.
} 
lo que permite que las barcas puedan moverse ágilmente bajo los puentes. Presentan una pendiente hacia el centro del vial lo que permite la evacuación de aguas de lluvia al nivel inferior a través de unas rejillas ubicadas en el suelo, que también permiten la entrada de luz y la ventilación (Firpo, 1963).

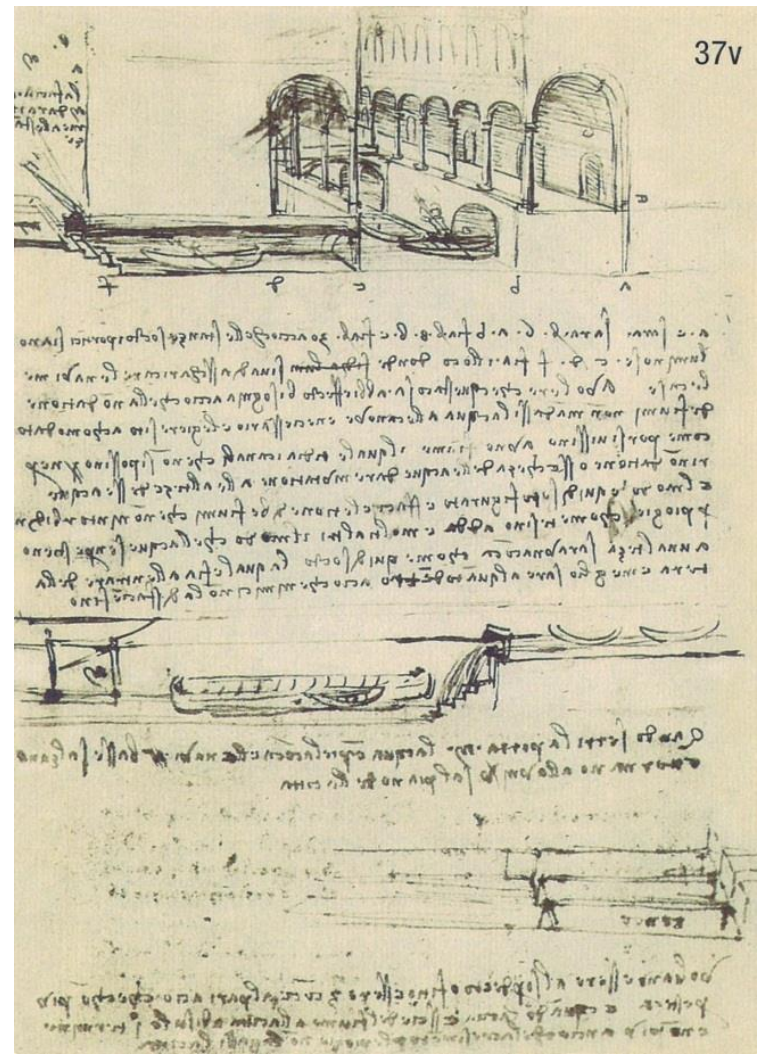

Fig. 2. Leonardo da Vinci. Ciudad ideal. Manuscrito B, folio 37v.

El propio Leonardo indica:

Por las calles altas no deben pasar carros, ni cosas semejantes, sino solamente los gentiles hombres. Por las calles bajas deberán ir los carros y otras bestias de carga, para uso y comodidad del pueblo. Cada cosa deberá dar la espalda a otra, dejando la calle baja en medio (Franchetti, 1985: 570).

Es interesante como Leonardo contempla que los pórticos de las viviendas den directamente a los canales para así poder llevar las barcas con personas y mercancías hasta la base del edificio. Leonardo indica: "Por las calles subterráneas circularán carruajes, zonas de establos y cosas fétidas. La distancia entre 
arcos será de 300 brazos y cada camino recibirá la luz de las grietas de las calles arriba" (Firpo, 1963: 75, trad. D. Hidalgo). Seguramente que Leonardo y a nivel arquitectónico pensó en viviendas con escaleras hasta el sótano, de tal forma que se pudiera acceder hasta los canales. De esta forma se podría realizar el transporte de personas y mercancías desde el interior de la vivienda, facilitando los procesos de carga y descarga. Leonardo establece:

La cara a-m dará la luz a la estancia; a-e estará a seis brazos, a-b sea brazos abajo, bc sea treinta brazos, para que por debajo los pórticos sean luminosos; c d f sea el lugar donde se van a descargar las barcas en las viviendas. Para que esta distribución sea practicable y que la inundación de los ríos no pueda penetrar en las bodegas, es preciso elegir una situación adecuada, como un lugar cerca de un río que pueda desviarse en canales en los que el nivel del agua no varíe en caso de inundaciones no de sequía. La construcción se muestra abajo; y elija un buen río, que no se enturbie con las lluvias, como el Ticino, el Adda y muchos otros. La construcción para forzar un nivel constante de las aguas será una especie de dársena situada a la entrada de la ciudad; o mejor aún, ya dentro de los límites de la ciudad, para que el enemigo no pueda destruirla (Firpo, 1966: 70, trad. D. Hidalgo).

En el folio 37r (véase fig. 3), aparece un resumen de los distintos elementos del tejido urbano: nivel superior con edificios y calles peatonales; nivel inferior que incluye calles de transporte y canales. En la representación, las bodegas de los talleres se encuentran indicadas con las letras $b$ y $c$, con la letra $d$ los tribunales y las escaleras de los puntos de conexión, con $e$ una calle baja, o quizás también un canal a cielo abierto cruzado por un puente (Firpo, 1963).

En el folio 15v, (véase fig. 4), se puede apreciar un edificio con torreones en las esquinas, rodeado por un foso en el que desembocan los canales por grandes arcadas. Se observa la red ortogonal de canales y dos rampas que permiten el acceso al edificio. En la parte inferior derecha aparece una ilustración con los dos niveles de comunicación y como Leonardo estudia y resuelve el enlace entre estos. Pero a su vez, establece relaciones métricas entre la anchura de las calles del nivel superior y la altura de los edificios colindantes. Para obtener una buena ventilación e iluminación de los viales peatonales, las calles deben tener una anchura igual o superior a los edificios ${ }^{16}$. Leonardo desarrolla la pro-

\footnotetext{
${ }^{16}$ De estos parámetros que regulan las condiciones de habitabilidad y ventilación ya existía el precedente de León Battista Alberti en su tratado De re aedificatoria.
} 
puesta de ciudad de tal forma que los viales presentan dimensiones iguales o superiores a la altura de las construcciones (Pedretti, 1988). También incluye grandes espacios públicos o zonas abiertas que permiten la respiración de la ciudad.
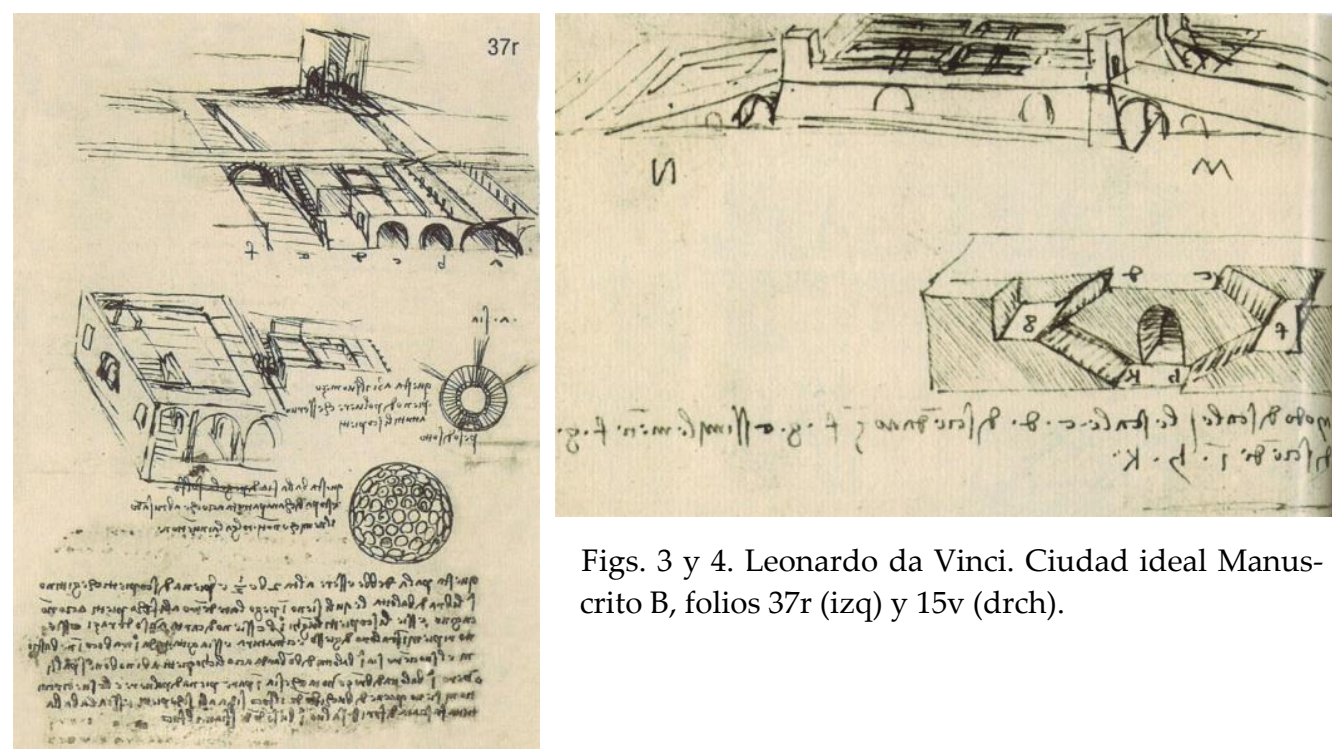

Figs. 3 y 4. Leonardo da Vinci. Ciudad ideal Manuscrito B, folios $37 \mathrm{r}$ (izq) y 15v (drch).

La descripción de Leonardo en relación a los viales:

Las calles m son 6 braccia más altas que las calles p-s, y cada calle debe tener 20 braccia de ancho y $1 / 2$ braccio de pendiente desde los bordes hacia el medio; $y$ en el centro, a intervalos de un braccio, deberá haber una abertura de un braccio de largo y un dedo de ancho, por donde pueda filtrarse el agua a huecos realizados al mismo nivel que donde pueda filtrarse el agua a huecos realizados al mismo nivel que p-s. A cada lado, en los extremos de la anchura de dicha calle, habrá una arcada, de 6 braccia de ancho, sobre columnas; debe entenderse que el que quiera pasear por toda la zona por las calles del nivel superior pueda usarlas para tal fin, mientras que el que desee ir por el nivel inferior pueda hacer lo mismo. Por las calles superiores no circularán vehículos ni objetos similares, sino que son para el uso exclusivo de los caballeros. Los carros y las cargas para el uso y comodidad de los habitantes deberán utilizar las calles inferiores. Las casas deben darse la espalda unas a otras, dejando las calles inferiores entre ellas. Los suministros, como la madera, el vino y otras cosas por el estilo, se llevarán por las puertas n, mientras que los retretes, establos y otras materias fétidas deberán vaciarse subterráneamente. De un arco al siguiente 
debe haber 300 braccia; cada calle recibirá luz por las aberturas de las calles superiores; en cada arco debe haber unas escaleras de caracol sobre un plano circular, porque los rincones de las cuadradas están siempre sucios. Deben ser anchas y, en la primera bóveda, debe haber una puerta de acceso a las letrinas públicas; dichas escaleras deben conducir de las calles superiores a las inferiores. Las calles superiores empiezan fuera de las puertas de la ciudad y suben en pendiente hasta que, en esas puertas, alcanzan una altura de 6 braccia. Esta ciudad debe construirse cerca del mar o de un gran río, para que las aguas puedan llevarse la suciedad de la ciudad (Anna, 2006: 230).

En el folio 16r, (véase fig. 5), aparece un espectacular palacio señorial donde se muestran los niveles indicados anteriormente. Leonardo nos complace con esta representación a vista de pájaro ${ }^{17}$ donde se puede apreciar el patio en planta baja que da acceso al canal y separado de este mediante un murete. La planta alta ubicada al nivel de las calles superiores también dispone de un patio. Todo el edificio se encuentra porticado y con una excelente decoración.

En los folios $68 \mathrm{v}$ y $47 \mathrm{r}$, (véase fig. 6), aparecen representados dos bloques de viviendas con escaleras dobles en el núcleo central del edificio. De esta forma, cada planta del edificio presenta un acceso independiente.

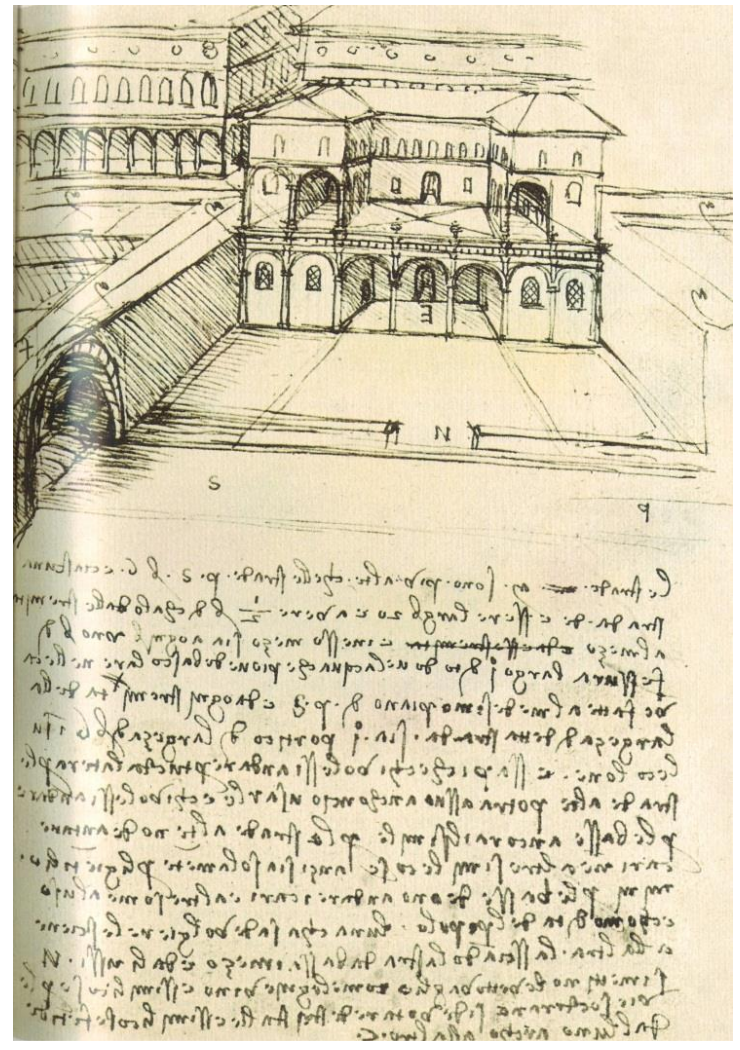

Fig. 5. Leonardo da Vinci, Ciudad ideal.

Manuscrito B, fol. 16r.

\footnotetext{
${ }_{17}$ Nombre designado por Wolfgang Lotz en su libro: La arquitectura del Renacimiento en Italia: Estudios. Con este término se refiere a la perspectiva muy habitual en los Códices de Leonardo donde la línea de horizonte se encuentra por encima de la línea de tierra y que permite obtener unas representaciones perspectivas en altura.
} 
En el folio $68 \mathrm{v}$, se aprecia claramente como el bloque de escaleras se encuentra en la parte central del edificio, motivado por la representación de líneas esquemáticas de las viviendas, que permiten la visualización completa del edificio. En el gráfico de la parte izquierda de la figura 6, se puede apreciar la distribución de una vivienda. El motivo por el cual solo se representa una vivienda es seguramente porque todas las viviendas cuentan con la misma distribución (Pedretti, 1988).
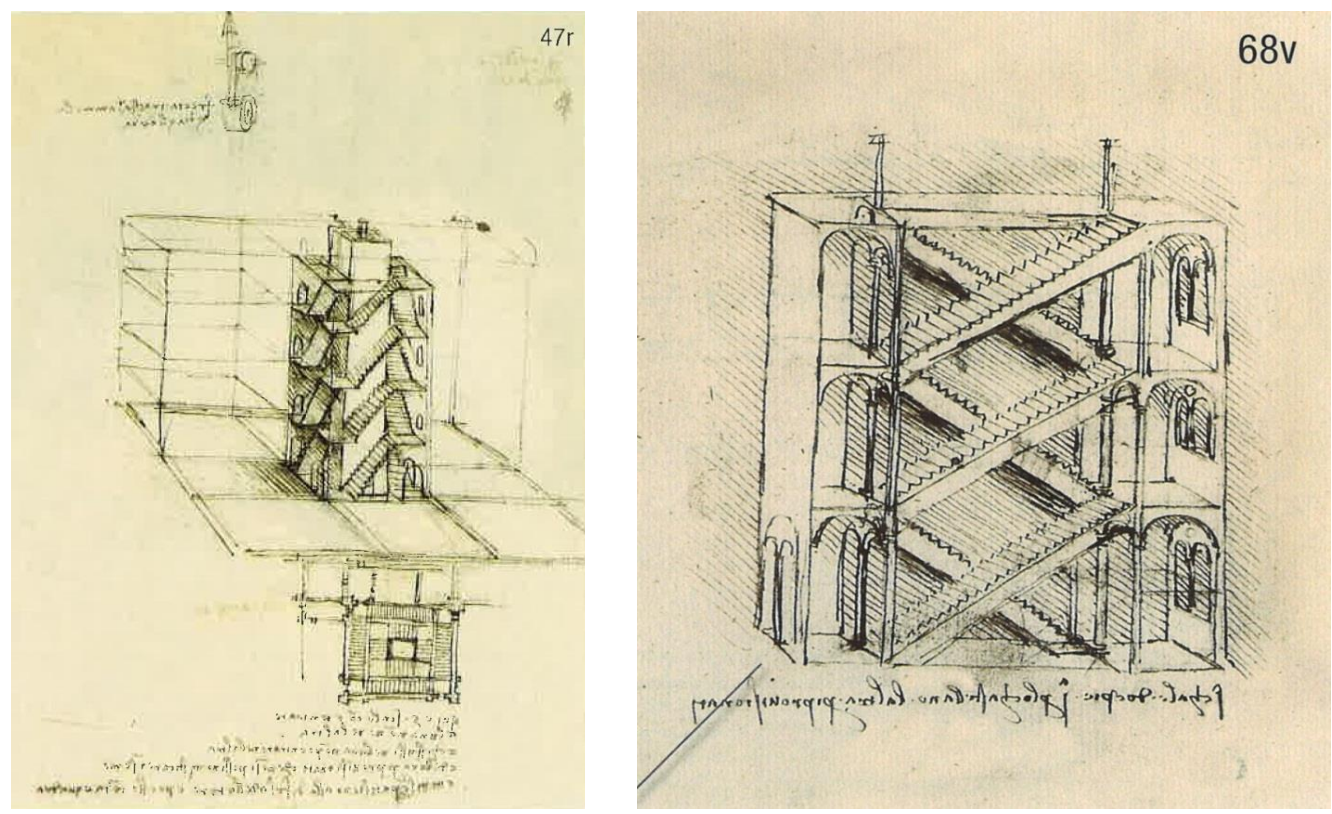

Fig. 6. Leonardo da Vinci, Ciudad ideal. Manuscrito B, folios 68v y 47r.

Algunos autores observan una posible estratificación social en la propuesta de dos niveles de la ciudad de Leonardo (Frnchetti, 1985). La justificación a esta diferenciación social es argumentada en que los dos niveles de circulación no son solamente físicos sino también sociales. En base a esa diferenciación, la nobleza podría andar por las calles superiores y por el nivel inferior solo las clases bajas. Examinada la propuesta de Leonardo no se aprecia en ella nada en relación a esta posible estratificación social de la población. El establecer dos niveles de circulación, uno superior y limpio y otro inferior, no es suficiente como para asegurar que Leonardo proponía una diferenciación social entre clases. En su propuesta de ciudad ideal no se observa, ni existe constancia escrita, de nin- 
gún tipo de vivienda subterránea para albergar a clases sociales más baja ${ }^{18}$. Este punto también es tratado por Franchetti aunque sus ideas y comentarios también desechan la teoría de una posible diferenciación entre clases

A modo de resumen, las ideas principales de este proyecto son:

$1^{\circ}$ ) Múltiples ciudades con capacidad máxima de 30.000 habitantes.

$\left.2^{\circ}\right)$ Ciudad sin amurallar.

3ํ) Circulación a distinto nivel: nivel superior abierto para tránsito de personas. Nivel inferior cerrado y para el tránsito de carros y mercancías.

$\left.4^{\circ}\right)$ Red de canales que permiten mejoras en el saneamiento y en el abastecimiento de la ciudad.

5º) Disposición de grandes espacios libres y abiertos.

6ํ) Regulación en las viviendas y calles de parámetros de ventilación e iluminación.

Esta propuesta fue presentada a Ludovico Sforza y no tiene un carácter utópico como las establecidas por otros autores de la época, más bien se trata de una ciudad construible ${ }^{19}$. La ciudad de Leonardo es perfectamente ejecutable y sus criterios se adelantan cuatrocientos años a la primera urbe construida de esta forma (Frere, 2001). No se tiene constancia que la propuesta llegara a realizarse. Posiblemente por la gran envergadura de la misma, el excesivo coste económico que supondría la realización o incluso la inestabilidad política que se vivía en ese momento en el Ducado de Milán y que podría originar una intervención militar en cualquier momento (Pedretti, 1988).

${ }^{18}$ El autor Firpo contradice esta teoría: El plano de las calles bajas no conserva nada de humillante ni de malsano: es simplemente el plano de los servicios, transportes y del trabajo, que se diferencia del plano de paseo, de la diversión, del culto, de la conservación (Firpo, 1963: 80, trad. D. Hidalgo).

${ }^{19}$ Cantone en relación a la opinión de Leonardo sobre el ideal clásico de la Antigüedad indica: Al proyectarla [ciudad ideal] Leonardo acepta el lenguaje arquitectónico del orden antiguo y lo aplica con la misma lógica empleada en sus invenciones científicas, subordinando lo estético a lo funcional (Cantone, 1978: 67, trad. D.Hidalgo). Por tanto y recuperando lo indicado en el capítulo anterior en relación a la idea y el gusto de Leonardo por el arte clásico, Cantone coincide con otros autores en afirmar que lo admiraba y lo aplicaba en sus estudios y propuestas de intervención. 


\section{Conclusiones.}

A Leonardo le interesaba la arquitectura, el urbanismo y la renovación urbana y en sus propuestas se observa la misma seriedad e ingenio que en otros estudios de otras áreas en las que es considerado como excepcional. La manifestación más paradigmática de la arquitectura es la ciudad. En ella se pueden observar claramente dos tipos de trabajos arquitectónicos: ordinarios y monumentales. Los primeros enfocados a viviendas y edificios de uso residencial y con una limitación temporal. Los segundos a sobrevivir con el paso del tiempo y dejar constancia de la grandeza de los imperios o de las épocas. Leonardo dejó constancia en sus manuscritos de una ciudad que cuenta con esos dos elementos arquitectónicos. Por un lado, su ciudad dispone de viviendas y edificios dignos para la población y por otro lado, dispone de unas características que debieran perdurar a lo largo de la historia y que expondrían al resto de Estados el esplendor del Ducado de Milán.

La cittá idéale está considerada como excepcional y de una brillantez majestuosa. Leonardo rompió con las teorías y propuestas de ciudades ideales del Renacimiento. Propuso una ciudad sin amurallar, sobre una red de canales que sirven como abastecimiento, transporte entre distintos puntos de la ciudad y para la evacuación de residuales. Dos niveles de circulación para carruajes, mercancías y peatones; Calles superiores amplias, soleadas y grandes zonas de esparcimiento e inferiores para las mercancías.

La propuesta se adelanta 400 años a su época y le otorga el reconocimiento de ser un urbanista excepcional. Así para Luigi Firpo è veramente il primo urbanista moderno (Firpo, 1963: 64), que implica no solo considerar a Leonardo como el promotor del cambio del urbanismo de la época y que posibilitó el desarrollo del urbanismo moderno, sino que disponía de las habilidades y conocimientos necesarios para ser tratado como un excepcional arquitecto, al igual que otros autores de la época de reconocimiento histórico. Esta connotación supondría no tener en cuenta como urbanistas a León Battista Alberti y Filarete cuyos tratados fueron escritos en los años 1452 y 1464 respectivamente. Esta exclusión viene justificada por algunos autores históricos al establecer el inicio de la edad moderna en el año 1492 coincidiendo con el descubrimiento de América. De esta forma lo indicado por Firpo podría interpretarse como que Alberti y Filarete fueron urbanistas pero no de la edad moderna, siendo Leonardo el primer urbanista a partir del año 1492. 


\section{Bibliografía.}

AA.VV. (2010): Il laboratorio di Leonardo nella cittá idéale. Editorial il laboratorio di Leonardo 3. Milano.

ALBERTI, León Battista (1991): De re aedificatoria, (traducción de Javier Fresni1lo). Editorial Akal. Madrid.

AnNA, H (2006): Leonardo da Vinci, cuadernos. Ediciones Parragón. Barcelona.

ARÉVAlO, Federico (2000): La representación de la ciudad en el renacimiento: levantamiento urbano y territorial. Tesis Doctoral dirigida por José María Gentil Baldrich. Escuela Técnica Superior de Arquitectura. Sevilla.

BENÉVOLO, Leonardo (1969): La cittá italiana del Rinascimento. Il polifilo. Milano.

BENÉVOLO, Leonardo (1978): Historia de la arquitectura del Renacimiento. Editorial Gustavo Gili. Barcelona.

Calatrava, J. et al (2010): Arquitectura escrita. Círculo de Bellas Artes, Madrid, pp. 105-111.

CAMPBELL, Erin (2008): “Leonardo da Vinci, Michelangelo ant the Renaissance in Florence". University of Toronto Quaterly. Toronto, vol. 77, no 1, pp 237-239.

CANTONE, G. (1978): La cittá di Marmo. Di Alberti a Serlio la storia tra progettazione e restauro. Oficina edizioni. Roma.

CLARK, Kenneth (2000): Leonardo da Vinci. Alianza editorial. Madrid.

CroiX, H. (1960): "Military architecture and the radial city plan in sixteenth century Italy". The art Bulletin. New Yotk, vol. 42, no 4, pp. 263-290.

DocCI, M. (2009): Storia dil rilevamento architettonico e urbano. Ed. Laterza. Roma.

FIRPO, Luigi (1963): Leonardo architteto e urbanista. Unione tipográfico- editrice Torinese. Turín.

FIRPO, Luigi (1975): La cittá ideales del Rinascimento urbanística e society. Unione tipográfico-editrice torinese. Turín.

FRANCHETTI, Vittorio (1985): Historia del urbanismo: Siglos XIV y XV. Instituto de estudios de administración local. Madrid.

FRERE, Jean Claude (2001): Leonardo da Vinci. Ediciones Lisma. Madrid.

FuENTE, M. J. (1991): “La Ciudad de Dios. El factor religioso en la construcción de la ciudad medieval”. Historia 16, XVI, no 186, pp. 51-60.

GARIN, Eugenio (1972): La cittá in Leonardo. Barberá. Firenze.

GIORGIO, F. (1967): Códice Magiliabechiano, tomo II, (edición consultada: Tratatti di architettura ingegneria e arte militare, vol I y II. Ediciones Maltese. Milán). 
GIORGIO, F. (1967): Códice Saluzziano, tomo II, (edición consultada: Tratatti di architettura ingegneria e arte militare, vol I y II. Ediciones Maltese. Milán).

GUIDONI, Enrico. (1978): La cittá europea. Electa. Milán.

LOTZ, Wolfgang (1985): La arquitectura del Renacimiento en Italia: Estudios, Biblioteca Básica de Arquitectura. Hermann Blume. Madrid.

MORENO, Evelio (1991): Las ciudades ideales del siglo XVI. Sendai. Madrid.

MORENO, Bibiana (2001): Artistas y artesanos del Barroco granadino. Documentación y estudio histórico de los gremios. Universidad de Granada. Granada.

MORRIS, A. (2007): Historia de la forma urbana. Gustavo Gili. Barcelona.

MRAKIC, Alessandro (2013): El desarrollo del conocimiento constructivo militar. Desde Vitruvio hasta el siglo XVII. Tesis doctoral dirigida por Manuel Martínez Blanco. Universidad Politécnica de Valencia. Valencia.

MURATORE, Giorgio (1980): La ciudad renacentista: tipos y modelos a través de los tratados. Instituto de Estudios Locales. Madrid.

PEDRETTI, Carlo (1988): Leonardo Architetto. Electa. Milán.

PUPPI, L. (1973): “la cittá ideale nella cultura architettonica del Rinascimento europeo". Atti XXII Congresso Internazionale di Storia dell'Arte. Budapest, pp. 649-658.

SIMONCINI, Giorgio (1974): Cittá e societá nel Rinascimento. Einaudi. Turín.

SISI, Enrico (1956): L`urbanística negli studi di Leonardo da Vinci. Tipografía editrice. Florencia.

SCAMOZZI, Francesco (1615). L'idea dell architettura universale. Biblioteca del Hospital Real de Granada.

SETA, Cesare (2002): La ciudad europea del siglo XV al XX. Istmo. Madrid.

TAglialagAmBA, Sara (2010): LeonardoEL'Arquitectura. CB Edizioni. Poggio a Caiano.

VAGNETTI, Luigi (1974): L ' architecto nella storia di occidente. Teorema. Florencia.

VERA, A (2010): La arquitectura militar del Renacimiento a través de los tratados de los siglos XV y XVI. Tesis doctoral dirigida por Juan Noguera Giménez. Universidad Politécnica de Valencia. Valencia.

VitruVIO, Marco (1999): X Libros de Arquitectura (Traducción de Lázaro de Velasco). Cicón ediciones. Caceres.

ZOLLNER, F. (2007). Leonardo da Vinci: obra pictórica completa y obra gráfica. Taschen. Colonia. 